\title{
The Effect of Maple Syrup Ingestion on Fat Oxidation During Incremental Exercise in Endurance Athletes
}

\author{
Satoshi Hattori ${ }^{1}$, Ayaka Noguchi ${ }^{2}$, Hitomi Ogata ${ }^{1,3}$, Masashi Kobayashi ${ }^{2}$, Naomi Omi ${ }^{1,}$ * \\ ${ }^{1}$ Faculty of Health and Sport Sciences, University of Tsukuba, Tsukuba, Japan \\ ${ }^{2}$ Comprehensive Human Sciences, University of Tsukuba, Tsukuba, Japan \\ ${ }^{3}$ Research Fellowship for Young Scientists, Japan Society for the Promotion of Science, Tokyo, Japan
}

Email address:

omi.naomign@u.tsukuba.ac.jp (N. Omi)

${ }^{*}$ Corresponding author

\section{To cite this article:}

Satoshi Hattori, Ayaka Noguchi, Hitomi Ogata, Masashi Kobayashi, Naomi Omi. The Effect of Maple Syrup Ingestion on Fat Oxidation During Incremental Exercise in Endurance Athletes. American Journal of Sports Science. Vol. 7, No. 4, 2019, pp. 149-154. doi: 10.11648/j.ajss.20190704.13

Received: September 28, 2019; Accepted: October 24, 2019; Published: October 30, 2019

\begin{abstract}
Maple syrup (MAP) is derived from the inspissation of acer saccharum sap, and this main material is composed as a sucrose-like carbohydrate (CHO). This food substance has metabolic effects on mammalian cells and some animal models. We hypothesized MAP ingestion would enable athletes to carry out endurance exercise without inhibiting fat oxidation. Here we investigate the effect of MAP ingestion on fat oxidation during incremental exercise on a cycle ergometer in endurance athletes $(\mathrm{n}=10)$ who exercised after ingesting MAP or sucrose (SUC). We measured fat and CHO oxidation, blood glucose concentration, and blood lactate concentration of subjects during incremental exercise. Between MAP and SUC groups, average fat and $\mathrm{CHO}$ oxidation was significantly different $(\mathrm{p}<0.01$ and $\mathrm{p}<0.001$, respectively). Blood lactate concentrations in the MAP group were significantly lower than in the SUC group, $-5,0 \mathrm{~min}(\mathrm{p}<0.05)$. In addition, blood glucose concentration in the SUC group at 24 min was significantly lower than at $-5 \min (\mathrm{p}<0.05)$. Our results indicated that MAP ingestion promoted only slight fat oxidation and a slow increase in blood lactate concentration compared with sucrose ingestion.
\end{abstract}

Keywords: Maple Syrup, Endurance Exercise, Fat Oxidation, Carbohydrate Oxidation

\section{Introduction}

Exercise inputs affect many biological contexts through signals to physiological tissues. In particular, skeletal muscle not only takes on metabolic changes but also works as a "mediator" to produce a massive impact on physiological circumstances. Therefore, exercise inhibits various health problems, especially in the cluster of conditions known as metabolic syndrome. Exercise is known to be an excellent preventive method against serious health problems such as hypertension, coronary artery disease, and type 2 diabetes [1]. Notably, endurance training is superior to other kinds of exercise in terms of suppressing these diseases [2].

Lipid droplets form a large energy substrate of adipose tissue. A marked accumulation of lipids leads to the pathological conditions of metabolic syndrome. Therefore, fat oxidation is known to be one of the key parameters for maximizing the effects of exercise to solve health problems. A lifestyle that neglects a healthy diet and exercise leads to a differentiation of mature adipocyte from preadipocyte. Mature adipocytes induce insulin resistance via cell-signaling proteins such as tumour necrosis factor (TNF)- $\alpha$ [3]. On the other hand, endurance exercise mainly mobilizes the energy substrates to mitochondria from adipose tissue. Because fat oxidation is dependent on exercise intensity in an inverted U-shaped curve, it peaks at $60 \%$ $\mathrm{VO}_{2 \max }$ [4]. This fat oxidation value is called "Fat $\max _{\max }$ [5]. Therefore, it is important to propose Fat ${ }_{\max }$ as a therapeutic intervention to metabolize lipid droplets in adipose tissue during exercise.

People often drink fluids containing a carbohydrate (CHO) during exercise training. Such ingestion is useful for maintaining endurance performance and inhibiting $\mathrm{CHO}$ depletion in the skeletal muscle and brain. However, such drinks reduce fat oxidation as opposed to promoting $\mathrm{CHO}$ oxidation during 
exercise. Thus, the ideal drink would supply $\mathrm{CHO}$ substrate to some organs, but not inhibit fat oxidation in exercise training.

Maple syrup may be a CHO-containing food material that does not inhibit fat oxidation during endurance exercise. It is made by inspissation of acer saccharum sap, and is composed of a CHO like sucrose. Moreover, maple syrup has a metabolic influence on physiological functions via abscisic acid (ABA), a phytohormone thought to exert metabolic effects in mammalian cells [6]. In fact, it reverses metabolic disease in obese mice by improving glucose tolerance and obesity-related inflammation [6]. Therefore, it is likely to support endurance exercise without leading to inhibition of fat oxidation.

The purpose of this study is to investigate the effect of a drink containing maple syrup on fat oxidation during endurance exercise. If our hypothesis is proved, we are likely to suggest a new carbohydrate material for a drink during endurance exercise.

\section{Methods}

\subsection{Subjects}

The subjects were endurance athletes (long distance runners and triathletes) with a mean \pm standard deviation age of $20.6 \pm 2.5$ yrs. They were recruited to participate in this study and provided written informed consent. This study was approved by the Ethics Committee of the University of Tsukuba. None of the subjects had a pathological condition and none were taking any medications or supplements.

\subsection{Preliminary Training}

Subjects were familiarized with the equipment and procedures before the start of the experiments. Approximately 1 week before the experimental trials, subjects were able to try a pedaling exercise on the electromagnetically braked cycle ergometer (PowerMax-VII, Combi Wellness, Tokyo).

Table 1. The physiological parameters of the subjects.

\begin{tabular}{ll}
\hline Physiological parameters & \\
\hline Age & $20.6 \pm 2.5$ \\
Height $(\mathrm{cm})$ & $172.3 \pm 5.2$ \\
Body weight $(\mathrm{kg})$ & $57.7 \pm 4.6$ \\
Body mass index $\left(\mathrm{kg} / \mathrm{m}^{2}\right)$ & $19.4 \pm 0.9$ \\
Body fat $(\%)$ & $10.6 \pm 2.1$ \\
$\dot{V} O_{2} \max (\mathrm{mL} / \mathrm{kg} / \mathrm{min})$ & $62.7 \pm 5.8$ \\
HRmax $($ beat $/ \mathrm{min})$ & $174.3 \pm 18.7$ \\
\hline
\end{tabular}

Values are mean \pm SD.

$\mathrm{VO}_{2}$ max: maximum oxygen uptake, HR: Heart rate.

\subsection{Prestudy Evaluation}

Subjects' body fat was measured using an impedance body composition analyzer (MC-190 SV, TANITA).

To evaluate subjects' maximum oxygen uptake $\left(\mathrm{VO}_{2 \max }\right)$, they performed a $\mathrm{V} \mathrm{O}_{2 \max }$ test on the electromagnetically braked cycle ergometer at least 1 week before the main experiment [7]. For the maximum cycling exercise test, subjects began with a 5 -min warm-up at $1 \mathrm{kp} / 60 \mathrm{rpm}$. The first stage of the exercise was at $2.5 \mathrm{kp}$ for $3 \mathrm{~min}$; $\mathrm{kp}$ was then increased by 0.1 every $1 \mathrm{~min}$ until exhaustion. Pedaling rate was set at $60 \mathrm{rpm}$ for all stages. During the test, subjects' heart rate (HR) was recorded continuously with an HR monitor (S610i, Polar, Tokyo). Breath-by-breath measurements were performed throughout the test using an aero-monitoring system (AE310-S, Minato Mẹdical Science Co., Tokyo).

Breath gas data $\left(\mathrm{VO}_{2}\right.$ and $\left.\mathrm{VCO}_{2}\right)$ were averaged for the last $1 \mathrm{~min}$ of each stage. Before each test, the gas analyzers were calibrated with gases of known concentrations $\left(5 \% \mathrm{CO}_{2}\right.$ and $15 \% \mathrm{O}_{2}$ ), and the volume was automatically calibrated at a flow rate of $2 \mathrm{~L} \cdot \mathrm{s}^{-1}$. The oxygen uptake $\left(\mathrm{V} \mathrm{O}_{2}\right)$ was considered to be maximal when two of the following three criteria were met: (1) a leveling of $\mathrm{VO}_{2}$ (defined as an increase of no more than $2 \mathrm{ml} \cdot \mathrm{kg}^{-1} \cdot \mathrm{min}^{-1}$ ) during the latter stages of the exercise test; (2) an HR value exceeding $90 \%$ of the age-predicted maximal value; and (3) a respiratory exchange ratio (RER) greater than 1.1. The laboratory room temperature was kept at $22 \pm 1^{\circ} \mathrm{C}$ during testing.

\subsection{Diets and Physical Activity the Day Before Testing}

We provided the subjects' diets the day before testing in order to ensure they complied with individual energy and nutrition balances based on dietary reference intakes for Japanese [8]. The diets were determined so as to achieve an individual energy balance, assuming the subject's resting metabolic rate to be $24.0 \mathrm{kcal} \cdot \mathrm{kg}^{-1} \cdot \mathrm{day}^{-1}$, and his physical activity factor to be 1.75 . The composition of the diets was $15 \%$ protein, $25 \%$ fat and $60 \%$ CHO. Physical activity on that day was confined to a normal level, and the subjects were instructed to not engage in intensity training.

\subsection{Experimental Design}

The study was a crossover design with random assignment of subjects, including the two exercise sessions and a washout period of at least 1week between experiments. All 10 subjects completed two exercise sessions: one after MAP ingestion, and the other after SUC ingestion. All exercise tests were performed in the morning, at the same time so as to avoid circadian variance. Subjects came to the laboratory on two occasions after fasting for 10- to 12-h overnight (since dinner the previous day). When subjects arrived at the laboratory, they were equipped with a heart rate monitor before drinking a beverage containing $8 \%$ SUC or $8 \%$ MAP per $500 \mathrm{ml}$ water, 15 min before the start of exercise in each experiment.

The incremental exercise protocol was based on Chenevière's test to estimate fat oxidation kinetics [9]. Briefly, subjects began with a 5 -min warm-up $(0.7 \mathrm{kp}, 60 \mathrm{rpm})$ on a cycle ergometer. Thereafter, the work rate was increased by $0.3 \mathrm{kp}$ every $3 \mathrm{~min}$ until $\mathrm{RER}=1.0$ without a rest period. The pedaling rate was set at $60 \mathrm{rpm}$ for all stages. Breath samples were collected with an aero-monitoring system during the incremental exercise after gas analyzers were calibrated. The breath-gas data $\left(\mathrm{VO}_{2}, \mathrm{VCO}_{2}\right)$ for the last $1 \mathrm{~min}$ of each stage were averaged. A blood sample $(150 \mu \mathrm{l})$ was collected using a Safety Lancet (Becton, Dickinson, Tokyo) and a capillary tube (Terumo, Tokyo) during the last 15 
s of each stage.

\subsection{Analysis}

The blood samples were analyzed immediately. Blood glucose concentrations were determined using a small electrodetype glucose analyzer (Antsense III, Horiba, Fukuoka, Japan); blood lactate concentrations were measured with a lactate analyzer (1500 Sport, YSI, Yellow Springs, OH).

\subsection{Calculations}

We calculated the total $\mathrm{CHO}$ and fat oxidation rates from the average values of $\mathrm{VO}_{2}$ and $\mathrm{CO}_{2}(\mathrm{~L} / \mathrm{min})$ during the last minute of every stage until RER $=1.0$. These were calculated using stoichiometric equations [10].

Fat oxidation rate $\left(\mathrm{g} \cdot \mathrm{min}^{-1}\right)=1.67 \dot{\mathrm{VO}}_{2}-1.67 \dot{\mathrm{VCO}}_{2}$

$$
\text { CHO oxidation rate }\left(\mathrm{g} \cdot \mathrm{min}^{-1}\right)=4.55 \dot{\mathrm{VCO}}_{2}-3.21 \dot{\mathrm{VO}}_{2}
$$

We used the SIN model to estimate fat oxidation kinetics during incremental exercise. The SIN model estimates fat oxidation kinetics based on the calculation of three independent variables (dilatation, symmetry, and translation) [9]. We simultaneously calculated three characteristics of the fat oxidation kinetics (i.e., the maximum fat oxidation [MFO]), which is the maximum fat oxidation during incremental exercise, the $\mathrm{Fat}_{\max }$ (exercise intensity at the MFO) [11], and the $\mathrm{Fat}_{\text {min }}$ (exercise intensity at the minimum value of fat oxidation).

\subsection{Statistics}

Experimental data were expressed as means \pm SD. Statistical analyses were calculated using SPSS statistical software (vers. 20, IBM Japan, Tokyo). A comparison of variables between two conditions was performed by student's $t$ test for paired samples. One-way analysis of variance (ANOVA) was used to test for statistically significant differences among times. The Holm's post hoc test was used for two-way ANOVA using R (vers. 3.6.1, R Development Core Team, Vienna, Austria). If a significant difference was detected among times, these groups were further evaluated using the post hoc Tukey test. $P<0.05$ was considered significant for all tests.

\section{Results}

\subsection{Subject Characteristics}

The physical characteristics of subjects are summarized in Table 1. The maximal performance values of subjects (i.e., $\mathrm{V}$ $\mathrm{O}_{2 \max }$ and $\mathrm{HR}_{\max }$ ) were determined in a prestudy evaluation.

\subsection{Fat Oxidation}

We investigated the influence of SUC or MAP ingestion on fat oxidation in endurance athletes. The results demonstrated a significant difference in average fat oxidation between groups $(p<0.01$, Figure $1 A)$. There were not significant differences in the iAUC of the fat oxidation in either group (Figure 1B), or at each time point in fat oxidation in groups. The results of two-way ANOVA were as follows: Beverage (not significant, n.s.), Time $(p<0.001)$, Bevarage $x$ Time interaction (n.s.). (Figure 1C). On the other hand, differences in fat oxidation kinetics were not significant (Dilatation, Translation, Symmetry), MFO, and Fat $_{\text {max }}$ between groups.

Table 2. Independent variables of the SIN model and characteristics of fat oxidation kinetics.

\begin{tabular}{lll}
\hline & SUC & MAP \\
\hline Dilatation & $-0.10 \pm 0.25$ & $-0.11 \pm 0.48$ \\
Translation & $-0.08 \pm 0.16$ & $1.05 \pm 0.02$ \\
Symmetry & $1.05 \pm 0.02$ & $1.06 \pm 0.01$ \\
MFO & $0.20 \pm 0.12$ & $0.26 \pm 0.12$ \\
Fatmax & $39.7 \pm 12.5$ & $54.9 \pm 19.2$ \\
\hline
\end{tabular}

Values are mean \pm SD. MFO: Maximam fat oxidation during incremental exercise. Fat $t_{\text {max }}$ : exercise intensity at the MFO.
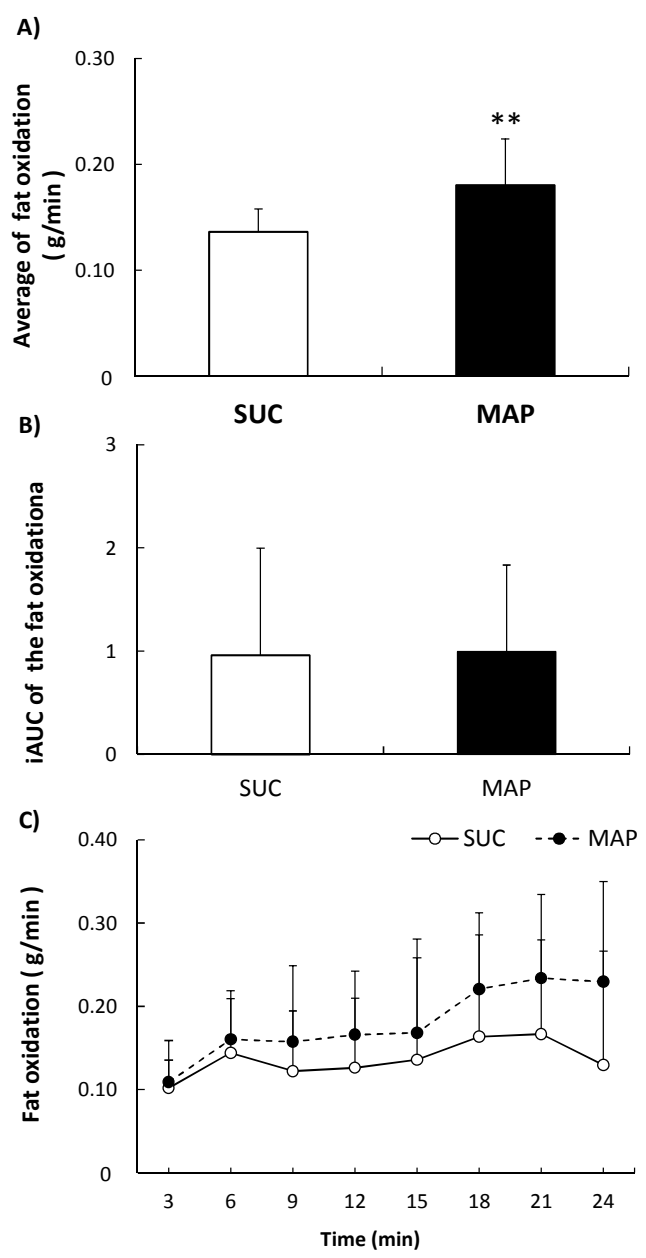

Figure 1. Fat oxidation levels during incremental exercise.

Average (A), and iAUC (B) of fat oxidation, (C) fat oxidation at time points during incremental increase in exercise. SUC (sucrose group), MAP (maple syrup group). Values are mean \pm SD. ${ }^{* *} \mathrm{p}<0.01$ SUC group vs. MAP group. The results of two-way ANOVA were as follows: Beverage (n.s.), Time $(\mathrm{p}<0.001)$, Beverage $\mathrm{x}$ Time interaction (n.s.) 


\subsection{CHO Oxidation}

We also investigated the influence of SUC or MAP ingestion on $\mathrm{CHO}$ oxidation in endurance athletes. Average $\mathrm{CHO}$ oxidation in the MAP group was higher than in the SUC group $(p<0.001$, Figure $2 A)$, and there were significant differences in the iAUC of $\mathrm{CHO}$ oxidation in both groups $(\mathrm{p}<0.05$, Figure 2B). Differences in fat oxidation at 3, 6, 9, $12,15-\mathrm{min}$ time points in both groups were significant $(p<0.05)$, and the results of two-way ANOVA were as follows: Beverage $(p<0.001)$, Time $(p<0.001)$, Beverage $x$ Time interaction (n.s.) (Figure 2C).

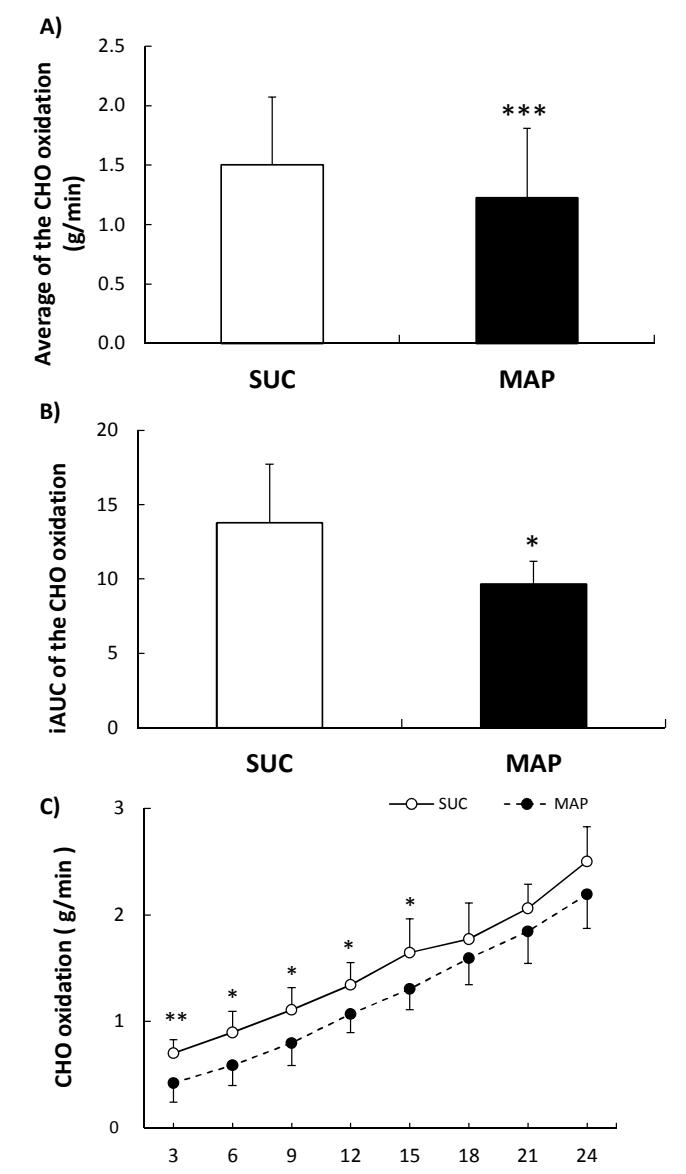

Figure 2. CHO oxidation levels during incremental exercise.

Average (A), and iAUC (B) of $\mathrm{CHO}$ oxidation, (C) $\mathrm{CHO}$ oxidation at time points during incremental increase in exercise. SUC (sucrose group), MAP (maple syrup group). Values are means \pm SD. ${ }^{*} \mathrm{p}<0.05,{ }^{* *} \mathrm{p}<0.01$ SUC group vs. MAP group. The results of two-way ANOVA were as follows: Beverage $(\mathrm{p}<0.001)$, Time $(\mathrm{p}<0.001)$, Beverage $\mathrm{x}$ Time interaction (n.s.)

\subsection{Blood Lactate Concentration}

Blood lactate concentrations in the MAP group at $-5,0$ min were lower than in the SUC group (Figure 3A). Moreover, blood lactate concentrations in the MAP group at $24 \mathrm{~min}$ were higher than at $-5 \mathrm{~min}(\mathrm{p}<0.05)$, whereas there were no such differences in the SUC group (Figure $3 \mathrm{~A}$ ). The results of two-way ANOVA were as follows: Beverage (n.s.), Time $(\mathrm{p}<0.01)$, Beverage $\mathrm{x}$ Time interaction (n.s.).

\subsection{Blood Glucose Concentration}

There were no significant differences in blood glucose concentration between groups (Figure 3C). On the other hand, blood glucose concentrations in the SUC group at 24 min were significantly lower than at $-5 \mathrm{~min}$, whereas these did not change in the MAP group (Figure 2B). The results of two-way ANOVA were as follows: Beverage $(\mathrm{p}<0.05)$, Time $(p<0.001)$, Beverage $x$ Time interaction (n.s.).

A)

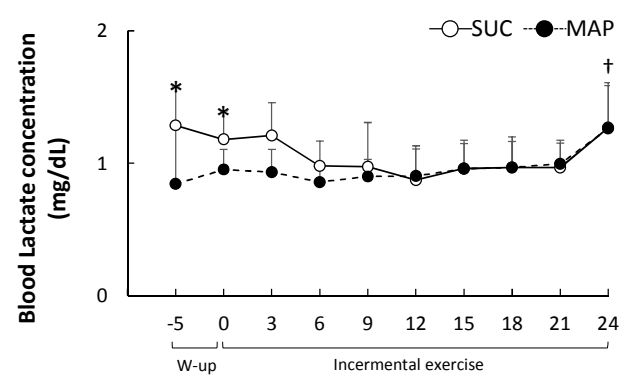

B)

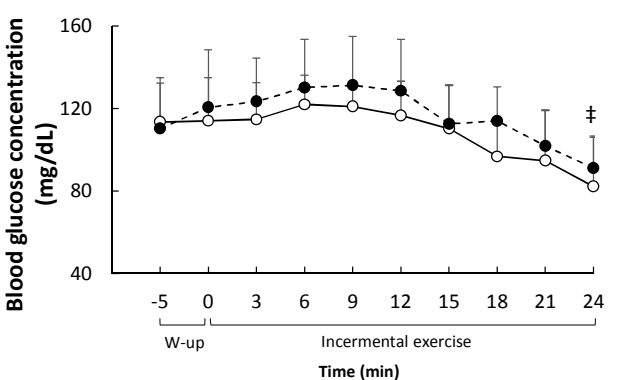

Figure 3. Blood lactate and glucose concentration during incremental exercise.

The blood lactate concentration (A) and blood glucose concentration (B) during incremental exercise in endurance athletes $(n=10)$. SUC (sucrose group), MAP (maple syrup group). $-5 \mathrm{~min}$; Rest period before w-up (warming up period), $-5 \mathrm{~min}$ to $0 \mathrm{~min}$; w-up, from $0 \mathrm{~min}$; incremental exercise period. Values are means \pm SD. ${ }^{*} \mathrm{p}<0.05$, SUC vs. MAP group. ${ }^{\dagger} \mathrm{p}<0.05,-5$ min vs. -24 min in MAP group. ${ }^{\ddagger} \mathrm{p}<0.05,-5$ min vs. -24 min in SUC group. The results of two-way ANOVA in blood lactate concentration were as follows: Beverage (n.s.), Time $(\mathrm{p}<0.01)$, Bevarage $X$ Time interaction (n.s.). The results of two-way ANOVA in blood glucose concentration were as follows: Beverage $(p<0.05)$, Time $(p<0.001)$, Beverage $x$ Time interaction (n.s.).

\section{Discussion}

We investigated the influence of MAP on fat and $\mathrm{CHO}$ oxidation, blood glucose concentration, and blood lactate concentration during incremental exercise. Primary results were that average fat oxidation in the MAP group was higher than in the SUC group. The average and iAUC of CHO oxidation were significantly lower in the MAP group compared to the SUC group. These results indicate that MAP ingestion did not strongly promote $\mathrm{CHO}$ oxidation and slightly inhibited fat oxidation compared with SUC during endurance exercise.

Average fat oxidation in the MAP group was higher than in the SUC group, while CHO oxidation in the MAP group was 
lower. It has been reported that ABA including MAP has some influence on energy metabolism [12]. ABA administration reversed abnormal energy metabolism in obese humans and animals [12]. Likewise, thiazolidinedione (structurally similar to ABA) up-regulated the $\beta$-oxidation and free fatty acid transport-related genes in adipose cells [13]. These findings indicated that MAP ingestion might influence fat metabolism through a pathway similar to ABA, although the detailed mechanisms remain to be clarified.

Blood lactate concentrations in the MAP group at $-5,0 \mathrm{~min}$ were lower than in the SUC group, while at 24 min, the MAP group had much higher concentration than at $-5 \mathrm{~min}$. In addition, blood glucose concentration in the SUC group at 24 min was lower than at $-5 \mathrm{~min}$, although the MAP group had no significant differences. $\mathrm{CHO}$ intakes usually up-regulate blood lactate concentration, whereas in our results this phenomenon was not observed in the MAP group. A previous study reported that blood glucose concentrations with MAP ingestion were lower than those for other natural sweeteners [14]. These results indicate that MAP enables the execution of an endurance exercise by maintaining the glycogen concentrations in organs.

There were no differences in the fat oxidation kinetics parameters. A previous study reported that these parameters were difficult between trained and untrained humans [9]. On the other hand, our study was used single subjects in a crossover protocol. It may be difficult to observe differences in the fat oxidation kinetics in both groups, because levels were low in terms of the influence of MAP ingestion on fat oxidation kinetics compared with a previous study.

One limitation of this study is that we did not confirm how MAP affected fat and $\mathrm{CHO}$ oxidation during incremental exercise. ABA, the main effecter of MAP, acts on energy metabolism via two independent roles: lipid accumulation as peroxisome proliferator-activated receptor $\gamma(\operatorname{PPAR} \gamma)$ ligand, and glucose uptake as lanthionine synthetase C-like 2 (LANCL2) ligand [15]. If these materials directly affect the skeletal muscle as a main energy consumer, they caused opposing results (decrease in fat oxidation and a promotion of CHO oxidation compared to the SUC group). Therefore, MAP-containing materials may indirectly influence the skeletal muscle through other organs. The results of this study suggest that MAP materials indirectly affect fat oxidation in skeletal muscle during exercise.

In conclusion, we revealed that MAP increased fat oxidation compared to SUC during endurance exercise. Moreover, it more decreased in CHO oxidation than SUC. In addition, MAP also slowed elevation of blood lactate concentrations and maintained blood glycogen levels compared to SUC. These results indicate that MAP ingestion as a food material promotes fat oxidation and weakly inhibits CHO oxidation compared with SUC ingestions.

\section{Conflict of Interest}

All the authours do not have any possible conflicts of interests.

\section{References}

[1] Pate RR, Pratt M, Blair SN, Haskell WL, Macera CA, Bouchard C, Buchner D, Ettinger W, Heath GW, King AC, et al.: Physical activity and public health. A recommendation from the Centers for Disease Control and Prevention and the American College of Sports Medicine. Jama 1995, 273: 402407.

[2] Donnelly JE, Blair SN, Jakicic JM, Manore MM, Rankin JW, Smith BK: American College of Sports Medicine Position Stand. Appropriate physical activity intervention strategies for weight loss and prevention of weight regain for adults. Med Sci Sports Exerc 2009, 41: 459-471.

[3] Hotamisligil GS, Shargill NS, Spiegelman BM: Adipose expression of tumor necrosis factor-alpha: direct role in obesity-linked insulin resistance. Science 1993, 259: 87-91.

[4] van Loon LJ, Greenhaff PL, Constantin-Teodosiu D, Saris $\mathrm{WH}$, Wagenmakers AJ: The effects of increasing exercise intensity on muscle fuel utilisation in humans. J Physiol 2001, 536: $295-304$

[5] Jeukendrup AE AJ: A new concept to optimize fat oxidation during exercise? Eur J Sports Sci 2001, 1 (5); 1-5.

[6] Guri AJ, Hontecillas R, Si H, Liu D, Bassaganya-Riera J: Dietary abscisic acid ameliorates glucose tolerance and obesity-related inflammation in $\mathrm{db} / \mathrm{db}$ mice fed high-fat diets. Clin Nutr 2007, 26: 107-116.

[7] Chin LM, Kowalchuk JM, Barstow TJ, Kondo N, Amano T, Shiojiri T, Koga S: The relationship between muscle deoxygenation and activation in different muscles of the quadriceps during cycle ramp exercise. J Appl Physiol (1985) 2011, 111: 1259-1265.

[8] Ministry of Health L, and Welfare of Japan: Dietary reference intakes for Japanese. Tokyo: Daiichi-Shuppan. 2005.

[9] Cheneviere X, Malatesta D, Peters EM, Borrani F: A mathematical model to describe fat oxidation kinetics during graded exercise. Med Sci Sports Exerc 2009, 41: 1615-1625.

[10] Frayn KN: Calculation of substrate oxidation rates in vivo from gaseous exchange. J Appl Physiol Respir Environ Exerc Physiol 1983, 55: 628-634.

[11] Brooks GA, Mercier J: Balance of carbohydrate and lipid utilization during exercise: the "crossover" concept. J Appl Physiol (1985) 1994, 76: 2253-2261.

[12] Sanchez-Sarasua S, Moustafa S, Garcia-Aviles A, LopezCliment MF, Gomez-Cadenas A, Olucha-Bordonau FE, Sanchez-Perez AM: The effect of abscisic acid chronic treatment on neuroinflammatory markers and memory in a rat model of high-fat diet induced neuroinflammation. Nutr Metab (Lond) 2016, 13: 73.

[13] Boden G, Homko C, Mozzoli M, Showe LC, Nichols C, Cheung P: Thiazolidinediones upregulate fatty acid uptake and oxidation in adipose tissue of diabetic patients. Diabetes 2005, 54: 880-885.

[14] St-Pierre P, Pilon G, Dumais V, Dion C, Dubois MJ, Dube P, Desjardins Y, Mrette A: Comparative analysis of maple syrup to other natural sweeteners and evaluation of their metabolic responses in healthy rats. J Funct Foods 2014, 11: 460-471. 
[15] Bruzzone S, Ameri P, Briatore L, Mannino E, Basile G, Andraghetti G, Grozio A, Magnone M, Guida L, Scarfi S, et al: The plant hormone abscisic acid increases in human plasma after hyperglycemia and stimulates glucose consumption by adipocytes and myoblasts. Faseb j 2012, 26: 1251-1260. 